Bull. Korean Math. Soc. 50 (2013), No. 3, pp. 787-799

http://dx.doi.org/10.4134/BKMS.2013.50.3.787

\title{
SECTIONAL SURVATURES OF THE SIEGEL-JACOBI SPACE
}

\author{
Jae-Hyun Yang, Young-Hoon Yong, Su-Na Huh, Jung-Hee Shin,
} AND GIL-Hong Min

\begin{abstract}
In this paper, we compute the sectional curvatures and the scalar curvature of the Siegel-Jacobi space $\mathbb{H}_{1} \times \mathbb{C}$ of degree 1 and index 1 explicitly.
\end{abstract}

\section{Introduction}

For a given fixed positive integer $n$, we let

$$
\mathbb{H}_{n}:=\left\{Z \in \mathbb{C}^{(n, n)} \mid Z={ }^{t} Z, \operatorname{Im} Z>0\right\}
$$

be the Siegel upper half plane of degree $n$ and let

$$
S p(n, \mathbb{R})=\left\{M \in \mathbb{R}^{(2 n, 2 n)} \mid{ }^{t} M J_{n} M=J_{n}\right\}
$$

be the symplectic group of degree $n$, where $F^{(k, l)}$ denotes the set of all $k \times l$ matrices with entries in a commutative ring $F$ for two positive integers $k$ and $l,{ }^{t} M$ denotes the transpose of a matrix $M, \operatorname{Im} Z$ denotes the imaginary part of $Z$ and

$$
J_{n}=\left(\begin{array}{cc}
0 & I_{n} \\
-I_{n} & 0
\end{array}\right) .
$$

Here $I_{n}$ denotes the $n \times n$ identity matrix. It is easy to see that $S p(n, \mathbb{R})$ acts on $\mathbb{H}_{n}$ transitively by

$$
M \cdot Z:=(A Z+B)(C Z+D)^{-1},
$$

where $M=\left(\begin{array}{cc}A & B \\ C & D\end{array}\right) \in S p(n, \mathbb{R})$ and $Z \in \mathbb{H}_{n}$.

For two positive integers $n$ and $m$, we consider the Heisenberg group

$$
H_{\mathbb{R}}^{(n, m)}:=\left\{(\lambda, \mu ; \kappa) \mid \lambda, \mu \in \mathbb{R}^{(m, n)}, \kappa \in \mathbb{R}^{(m, m)}, \kappa+\mu^{t} \lambda \text { symmetric }\right\}
$$

endowed with the following multiplication law

$$
(\lambda, \mu ; \kappa) \circ\left(\lambda^{\prime}, \mu^{\prime} ; \kappa^{\prime}\right):=\left(\lambda+\lambda^{\prime}, \mu+\mu^{\prime} ; \kappa+\kappa^{\prime}+\lambda^{t} \mu^{\prime}-\mu^{t} \lambda^{\prime}\right) .
$$

Received February 23, 2012; Revised May 22, 2012.

2010 Mathematics Subject Classification. Primary 32Q15, 32M10, 53C30.

Key words and phrases. Siegel-Jacobi space, sectional curvatures, scalar curvature.

This work was supported by Basic Science Program through the National Research Foundation of Korea(NRF) funded by the Ministry of Education, Science and Technology (43338$01)$. 
We define the semidirect product of $S p(n, \mathbb{R})$ and $H_{\mathbb{R}}^{(n, m)}$

$$
G_{n, m}^{J}:=S p(n, \mathbb{R}) \ltimes H_{\mathbb{R}}^{(n, m)}
$$

endowed with the following multiplication law

$$
(M,(\lambda, \mu ; \kappa)) \cdot\left(M^{\prime},\left(\lambda^{\prime}, \mu^{\prime} ; \kappa^{\prime}\right)\right):=\left(M M^{\prime},\left(\tilde{\lambda}+\lambda^{\prime}, \tilde{\mu}+\mu^{\prime} ; \kappa+\kappa^{\prime}+\tilde{\lambda}^{t} \mu^{\prime}-\tilde{\mu}^{t} \lambda^{\prime}\right)\right)
$$

with $M, M^{\prime} \in S p(n, \mathbb{R}),(\lambda, \mu ; \kappa),\left(\lambda^{\prime}, \mu^{\prime} ; \kappa^{\prime}\right) \in H_{\mathbb{R}}^{(n, m)}$ and $(\tilde{\lambda}, \tilde{\mu}):=(\lambda, \mu) M^{\prime}$.

We call this group $G_{n, m}^{J}$ the Jacobi group of degree $n$ and index $m$. It is easy to see that $G_{n, m}^{J}$ acts on $\mathbb{H}_{n} \times \mathbb{C}^{(m, n)}$ transitively by

$$
(M,(\lambda, \mu ; \kappa)) \cdot(Z, W):=\left(M \cdot Z,(W+\lambda Z+\mu)(C Z+D)^{-1}\right),
$$

where $M=\left(\begin{array}{cc}A & B \\ C & D\end{array}\right) \in S p(n, \mathbb{R}),(\lambda, \mu ; \kappa) \in H_{\mathbb{R}}^{(n, m)}$ and $(Z, W) \in \mathbb{H}_{n} \times \mathbb{C}^{(m, n)}$.

The homogeneous space $\mathbb{H}_{n} \times \mathbb{C}^{(m, n)}$ is called the Siegel-Jacobi space of degree $n$ and index $m$. We refer to $[3,5,6,7,9,10,11,12,13,14,15,16,17,18]$ for more details on materials related to the Siegel-Jacobi space.

In [14], the author proved that for any two positive real numbers $A$ and $B$, the following metric

$$
\begin{aligned}
d s_{n, m ; A, B}^{2}= & A \sigma\left(Y^{-1} d Z Y^{-1} d \bar{Z}\right) \\
+ & B\left\{\sigma\left(Y^{-1 t} V V Y^{-1} d Z Y^{-1} d \bar{Z}\right)+\sigma\left(Y^{-1 t}(d W) d \bar{W}\right)\right. \\
& \left.-\sigma\left(V Y^{-1} d Z Y^{-1 t}(d \bar{W})\right)-\sigma\left(V Y^{-1} d \bar{Z} Y^{-1 t}(d W)\right)\right\}
\end{aligned}
$$

is a Riemannian metric on the Siegel-Jacobi space $\mathbb{H}_{n} \times \mathbb{C}^{(m, n)}$ which is invariant under the action (1.2) of the Jacobi group $G_{n, m}^{J}$, where $Z=X+i Y \in \mathbb{H}_{n}, W=$ $U+i V \in \mathbb{C}^{(m, n)}$ with $Z=\left(z_{i j}\right), W=\left(w_{k l}\right)$ and $X, Y, U, V$ real, we put

$$
d Z=\left(d z_{i j}\right), \quad d \bar{Z}=\left(d \bar{z}_{i j}\right), \quad d W=\left(d w_{k l}\right), \quad d \bar{W}=\left(d \bar{w}_{k l}\right)
$$

and $\sigma(A)$ denotes the trace of a square matrix $A$. Also he computed the Laplace-Beltrami operator of the Siegel-Jacobi space $\left(\mathbb{H}_{n} \times \mathbb{C}^{(m, n)}, d s_{n, m ; A, B}^{2}\right)$ explicitly.

In this paper, we consider the case $n=1$ and $m=1$. In this case, we have a Riemannian metric

$$
\begin{aligned}
& d s_{1,1 ; A, B}^{2} \\
= & A \frac{d x^{2}+d y^{2}}{y^{2}}+B\left\{\frac{v^{2}}{y^{3}}\left(d x^{2}+d y^{2}\right)+\frac{1}{y}\left(d u^{2}+d v^{2}\right)-\frac{2 v}{y^{2}}(d x d u+d y d v)\right\}
\end{aligned}
$$

on $\mathbb{H}_{1} \times \mathbb{C}$ which is invariant under the action (1.2) of the Jacobi group $G_{1,1}^{J}=$ $S L(2, \mathbb{R}) \ltimes H_{\mathbb{R}}^{(1,1)}$, where $z=x+i y \in \mathbb{H}_{1}$ and $w=u+i v \in \mathbb{C}$ with $x, y, u, v$ real coordinates. We also refer to [1] and [4] for the metric (1.4). According to 
Theorem 1.2 in [14], we see that the Laplace-Beltrami operator $\Delta_{1,1 ; A, B}$ of the Siegel-Jacobi space $\left(\mathbb{H}_{1} \times \mathbb{C}, d s_{1,1 ; A, B}^{2}\right)$ is given by

$$
\begin{aligned}
\Delta_{1,1 ; A, B}= & \frac{1}{A}\left\{y^{2}\left(\frac{\partial^{2}}{\partial x^{2}}+\frac{\partial^{2}}{\partial y^{2}}\right)+v^{2}\left(\frac{\partial^{2}}{\partial u^{2}}+\frac{\partial^{2}}{\partial v^{2}}\right)\right. \\
& \left.+2 y v\left(\frac{\partial^{2}}{\partial x \partial u}+\frac{\partial^{2}}{\partial y \partial v}\right)\right\}+\frac{y}{B}\left(\frac{\partial^{2}}{\partial u^{2}}+\frac{\partial^{2}}{\partial v^{2}}\right) .
\end{aligned}
$$

The purpose of this paper is to compute the sectional curvatures of the SiegelJacobi space $\left(\mathbb{H}_{1} \times \mathbb{C}, d s_{1,1 ; A, B}^{2}\right)$ explicitly. We will prove that the scalar curvature $r(p)$ of $\left(\mathbb{H}_{1} \times \mathbb{C}, d s_{1,1 ; A, B}^{2}\right)$ is constant, precisely, $r(p)=-\frac{3}{A}$ for all $p \in \mathbb{H}_{1} \times \mathbb{C}$.

This paper is organized as follows. In Section 2, we compute the Christoffel symbols $\Gamma_{i j}^{k}$ of the Siegel-Jacobi space $\left(\mathbb{H}_{1} \times \mathbb{C}, d s_{1,1 ; A, B}^{2}\right)$ explicitly. In Section 3, we compute the sectional curvatures of the Siegel-Jacobi space $\left(\mathbb{H}_{1} \times \mathbb{C}, d s_{1,1 ; A, B}^{2}\right)$ explicitly. We prove that the scalar curvature of the SiegelJacobi space $\left(\mathbb{H}_{1} \times \mathbb{C}, d s_{1,1 ; A, B}^{2}\right)$ is given by $-\frac{3}{A}$ and that the scalar curvature is independent of the choice of $B$. In the final section, we discuss the invariant Riemannian metrics of the Siegel-Jacobi disk $\mathbb{D} \times \mathbb{C}$ and their Laplace-Beltrami operators.

Notations: We denote by $\mathbb{R}$ and $\mathbb{C}$ the field of real numbers, and the field of complex numbers respectively. The symbol ":=" means that the expression on the right is the definition of that on the left. For two positive integers $k$ and $l$, $F^{(k, l)}$ denotes the set of all $k \times l$ matrices with entries in a commutative ring $F$. For a square matrix $A \in F^{(k, k)}$ of degree $k, \sigma(A)$ denotes the trace of $A$. For any $M \in F^{(k, l)},{ }^{t} M$ denotes the transpose matrix of $M$. $I_{n}$ denotes the identity matrix of degree $n$.

\section{Preliminaries}

For brevity, we write $M:=\mathbb{H}_{1} \times \mathbb{C}$. Then $M$ is a four dimensional Riemannian manifold with a metric $d s^{2}$ given by (1.4). We denote by $C^{\infty}(M)$ and $\mathcal{X}(M)$ be the algebra of all $C^{\infty}$ functions on $M$ and the algebra of all $C^{\infty}$ vector fields on $M$ respectively. It is well known that there exists a uniquely determined Riemannian connection $\nabla$ on $M$ (cf. [2], p. 314). That is, the connection $\nabla$ is a mapping $\nabla: \mathcal{X}(M) \times \mathcal{X}(M) \longrightarrow \mathcal{X}(M)$, denoted by $\nabla(X, Y)=\nabla_{X} Y$ which satisfies the following properties (R1)-(R4): For all $f, g \in C^{\infty}(M)$ and $X, Y, Z, W \in \mathcal{X}(M)$,

$$
\begin{array}{ll}
(R 1) & \nabla_{f X+g Y} Z=f\left(\nabla_{X} Z\right)+g\left(\nabla_{Y} Z\right), \\
(R 2) & \nabla_{X}(f Y+g Z)=f\left(\nabla_{X} Y\right)+g\left(\nabla_{X} Z\right)+(X f) Y+(X g) Z, \\
(R 3) & {[X, Y]=\nabla_{X} Y-\nabla_{Y} X \text { (symmetry), and }} \\
(R 4) & X(g(Y, Z))=g\left(\nabla_{X} Y, Z\right)+g\left(Y, \nabla_{X} Z\right),
\end{array}
$$


where $g(Y, Z)$ denoted the inner product determined by the Riemannian metric $d s^{2}$ on $M$.

Now we fix a local cooodinate $x, y, u, v$ with $z=x+i y$ and $w=u+i v$. Then the smooth vector fields

$$
E_{1}:=\frac{\partial}{\partial x}, \quad E_{2}:=\frac{\partial}{\partial y}, \quad E_{3}:=\frac{\partial}{\partial u} \quad \text { and } E_{4}:=\frac{\partial}{\partial v}
$$

form a local frame fields on $M$. We recall that the Christoffel symbols $\Gamma_{i j}^{k}(1 \leq$ $i, j, k \leq 4)$ are defined by

$$
\nabla_{E_{i}} E_{j}:=\sum_{k=1}^{4} \Gamma_{i j}^{k} E_{k}, \quad 1 \leq i, j \leq 4 .
$$

According to (1.4), the matrix form $g=\left(g_{i j}\right)$ of the metric $d s_{1,1 ; A, B}^{2}$ is of the form

$$
g=\left(g_{i j}\right)=\left(\begin{array}{cccc}
\frac{A y+B v^{2}}{y^{3}} & 0 & -\frac{B v}{y^{2}} & 0 \\
0 & \frac{A y+B v^{2}}{y^{3}} & 0 & -\frac{B v}{y^{2}} \\
-\frac{B v}{y^{2}} & 0 & \frac{B}{y} & 0 \\
0 & -\frac{B v}{y^{2}} & 0 & \frac{B}{y}
\end{array}\right) .
$$

Then it is easy to see that $\operatorname{det}\left(g_{i j}\right)=A^{2} B^{2} y^{-6}$ and the inverse matrix $g^{-1}:=$ $\left(g^{i j}\right)$ of $g=\left(g_{i j}\right)$ is given by

$$
g^{-1}=\left(g^{i j}\right)=\left(\begin{array}{cccc}
\frac{y^{2}}{A} & 0 & \frac{y v}{A} & 0 \\
0 & \frac{y^{2}}{A} & 0 & \frac{y v}{A} \\
\frac{y v}{A} & 0 & \frac{A y+B v^{2}}{A B} & 0 \\
0 & \frac{y v}{A} & 0 & \frac{A y+B v^{2}}{A B}
\end{array}\right) .
$$

Lemma 2.1. For all $i, j, k, \Gamma_{i j}^{k}=\Gamma_{j i}^{k}$. The Christoffel symbols $\Gamma_{i j}^{k}$ 's $(1 \leq$ $i, j, k \leq 4)$ are given by

$$
\begin{array}{rlrl}
\Gamma_{11}^{2} & =\frac{2 A y+B v^{2}}{2 A y^{2}}, & \Gamma_{12}^{1}=\Gamma_{22}^{2}=-\frac{2 A y+B v^{2}}{2 A y^{2}} \\
\Gamma_{11}^{4}=\frac{B v^{3}}{2 A y^{3}}, & \Gamma_{12}^{3}=\Gamma_{22}^{4}=-\frac{B v^{3}}{2 A y^{3}} \\
\Gamma_{14}^{1}=\Gamma_{23}^{1}=\Gamma_{24}^{2}=\Gamma_{33}^{4}=\frac{B v}{2 A y}, & \Gamma_{13}^{2}=\Gamma_{34}^{3}=\Gamma_{44}^{4}=-\frac{B v}{2 A y}
\end{array}
$$




$$
\begin{array}{ll}
\Gamma_{13}^{4}=\frac{A y-B v^{2}}{2 A y^{2}}, & \Gamma_{14}^{3}=\Gamma_{23}^{3}=\Gamma_{24}^{4}=-\frac{A y-B v^{2}}{2 A y^{2}} \\
\Gamma_{33}^{2}=\frac{B}{2 A}, & \Gamma_{44}^{2}=\Gamma_{34}^{1}=-\frac{B}{2 A}
\end{array}
$$

and all other $\Gamma_{i j}^{k}=0$.

Proof. The first statement follows immediately from the symmetry relation (R3). We recall (cf. [2], p. 318 or [8], p. 210) that

$$
\Gamma_{i j}^{k}=\frac{1}{2} \sum_{s=1}^{4} g^{k s}\left(E_{j} g_{s i}-E_{s} g_{i j}+E_{i} g_{j s}\right)
$$

for all $i, j, k$. By an easy computation, we get all $\Gamma_{i j}^{k}$.

We define the functions

$$
h_{A}:=\frac{y^{\frac{3}{2}}}{\left(A y+B v^{2}\right)^{\frac{1}{2}}}, \quad h_{B}:=\frac{\sqrt{B} y v}{\sqrt{A}\left(A y+B v^{2}\right)^{\frac{1}{2}}}, \quad h_{C}:=\frac{\left(y+v^{2}\right)^{\frac{1}{2}}}{\sqrt{A B}} .
$$

An easy computation gives the following:

\section{Lemma 2.2.}

$$
\begin{array}{rlrl}
\frac{\partial h_{A}}{\partial y} & =\frac{y^{\frac{1}{2}}\left(2 A y+3 B v^{2}\right)}{2\left(A y+B v^{2}\right)^{\frac{3}{2}}}, & & \frac{\partial h_{B}}{\partial y}=\frac{\sqrt{B} v\left(A y+2 B v^{2}\right)}{2 \sqrt{A}\left(A y+B v^{2}\right)^{\frac{3}{2}}}, \\
\frac{\partial h_{C}}{\partial y}=\frac{\sqrt{A}}{2 \sqrt{B}\left(A y+B v^{2}\right)^{\frac{1}{2}}}, & \frac{\partial h_{A}}{\partial v}=-\frac{B y^{\frac{3}{2}} v}{\left(A y+B v^{2}\right)^{\frac{3}{2}}}, \\
\frac{\partial h_{B}}{\partial v}=\frac{\sqrt{A B} y^{2}}{\left(A y+B v^{2}\right)^{\frac{3}{2}}}, & \frac{\partial h_{C}}{\partial v}=\frac{\sqrt{B} v}{\sqrt{A}\left(A y+B v^{2}\right)^{\frac{1}{2}}}
\end{array}
$$

and

$$
\frac{\partial h_{A}}{\partial x}=\frac{\partial h_{B}}{\partial x}=\frac{\partial h_{C}}{\partial x}=\frac{\partial h_{A}}{\partial u}=\frac{\partial h_{B}}{\partial u}=\frac{\partial h_{C}}{\partial u}=0 .
$$

Lemma 2.3. The following frame field $F_{1}, F_{2}, F_{3}, F_{4}$ defined by

$$
\begin{array}{lll}
F_{1}:=h_{A} E_{1}, & F_{2}:=h_{A} E_{2} \\
F_{3}:=h_{B} E_{1}+h_{C} E_{3}, & F_{4}:=h_{B} E_{2}+h_{C} E_{4}
\end{array}
$$

form an orthonomal frame field on $M$. And they satisfy the following relations

$$
\begin{aligned}
& {\left[F_{1}, F_{2}\right]=-\frac{y^{2}\left(2 A y+3 B v^{2}\right)}{2\left(A y+B v^{2}\right)^{2}} E_{1}, \quad\left[F_{1}, F_{3}\right]=0,} \\
& {\left[F_{1}, F_{4}\right]=-\frac{B \sqrt{B} y^{\frac{3}{2}} v^{3}}{2 \sqrt{A}\left(A y+B v^{2}\right)^{2}} E_{1},} \\
& {\left[F_{2}, F_{3}\right]=\frac{\sqrt{B} y^{\frac{3}{2}} v\left(A y+2 B v^{2}\right)}{2 \sqrt{A}\left(A y+B v^{2}\right)^{2}} E_{1}+\frac{\sqrt{A} y^{\frac{3}{2}}}{2 \sqrt{B}\left(A y+B v^{2}\right)} E_{3},}
\end{aligned}
$$




$$
\left[F_{2}, F_{4}\right]=\frac{\sqrt{B} y^{\frac{3}{2}} v}{2 \sqrt{A}\left(A y+B v^{2}\right)} E_{2}+\frac{\sqrt{A} y^{\frac{3}{2}}}{2 \sqrt{B}\left(A y+B v^{2}\right)} E_{4}
$$

and

$$
\left[F_{3}, F_{4}\right]=-\frac{2 A^{2} y^{3}+3 A B y^{2} v^{2}+2 B^{2} y v^{4}}{2 A\left(A y+B v^{2}\right)^{2}} E_{1}-\frac{3 A y v+2 B v^{3}}{2 A\left(A y+B v^{2}\right)} E_{3} .
$$

Proof. The first statement follows from the Gram-Schmidt orthogonalization process. The proof of the second statement follows from a direct computation.

Definition 2.1. Let $X$ and $Y$ be two smooth vector fields on $M$. The curvature operator $R(X, Y): \mathcal{X}(M) \longrightarrow \mathcal{X}(M)$ is defined as

$$
R(X, Y) Z:=\nabla_{X}\left(\nabla_{Y} Z\right)-\nabla_{Y}\left(\nabla_{X} Z\right)-\nabla_{[X, Y]} Z, \quad Z \in \mathcal{X}(M) .
$$

For a quadruple $(\mathrm{X}, \mathrm{Y}, \mathrm{Z}, \mathrm{W})$ of smooth vector fields on $M$, we define

$$
R(X, Y, Z, W):=g(R(X, Y) Z, W) .
$$

The tensor $R(X, Y, Z, W)$ is called the Riemann curvature tensor of $M$.

\section{Sectional curvatures}

For any point $p \in M$, we let $\pi_{X, Y}$ be the plane section of tangent space $T_{p}(M)$ of $M$ at $p$ spanned by two orthonormal tangent vectors $X$ and $Y$ in $T_{p}(M)$. We recall that the sectional curvature $K_{p}\left(\pi_{X, Y}\right)$ of $\pi_{X, Y}$ is defined by

$$
K_{p}\left(\pi_{X, Y}\right):=-R(X, Y, Z, W)=-g(R(X, Y) Z, W),
$$

where $R(X, Y, Z, W)$ denotes the Riemann curvature tensor of $M$. In fact, the sectional curvature $K_{p}\left(\pi_{X, Y}\right)$ is independent of the choice of two orthonormal basis of the section $\pi_{X, Y}$.

Theorem 3.1. For any point $p=(x, y, u, v) \in M$, we let $\pi_{i j}$ the plane section of $T_{p}(M)$ spanned by two orthonormal vectors $F_{i p}$ and $F_{j p}$ of $T_{p}(M)$. Then the sectional curvatures $K_{p}\left(\pi_{X, Y}\right)$ are given by

$$
\begin{array}{lll}
K_{p}\left(\pi_{12}\right)=-\frac{1}{A}+\frac{3 B^{2} v^{4}}{2 A\left(A y+B v^{2}\right)^{2}}, & K_{p}\left(\pi_{13}\right)=-\frac{1}{4 A}, \\
K_{p}\left(\pi_{14}\right)=-\frac{1}{4 A}+\frac{3 A B y v^{2}}{2 A\left(A y+B v^{2}\right)^{2}}, & K_{p}\left(\pi_{23}\right)=-\frac{1}{4 A}+\frac{3 A B y v^{2}}{2 A\left(A y+B v^{2}\right)^{2}}, \\
K_{p}\left(\pi_{24}\right)=-\frac{1}{4 A}, & K_{p}\left(\pi_{34}\right)=\frac{1}{2 A}-\frac{3 B v^{2}\left(2 A y+B v^{2}\right)}{2 A\left(A y+B v^{2}\right)^{2}} .
\end{array}
$$

Proof. We observe that $K_{p}\left(\pi_{i j}\right)=-g\left(R\left(F_{i p}, F_{j p}\right) F_{i p}, F_{j p}\right)$ for $1 \leq i, j \leq 4$. By a direct computation, we obtain

$$
\begin{aligned}
& \nabla_{E_{1}} \nabla_{E_{2}} E_{1}=\left(\Gamma_{11}^{2} \Gamma_{12}^{1}+\Gamma_{12}^{3} \Gamma_{13}^{2}\right) E_{2}+\left(\Gamma_{11}^{4} \Gamma_{12}^{1}+\Gamma_{12}^{3} \Gamma_{13}^{4}\right) E_{4}, \\
& \nabla_{E_{1}} \nabla_{E_{2}} E_{2}=\left(\Gamma_{12}^{1} \Gamma_{22}^{2}+\Gamma_{14}^{1} \Gamma_{22}^{4}\right) E_{1}+\left(\Gamma_{12}^{3} \Gamma_{22}^{2}+\Gamma_{14}^{3} \Gamma_{22}^{4}\right) E_{3},
\end{aligned}
$$




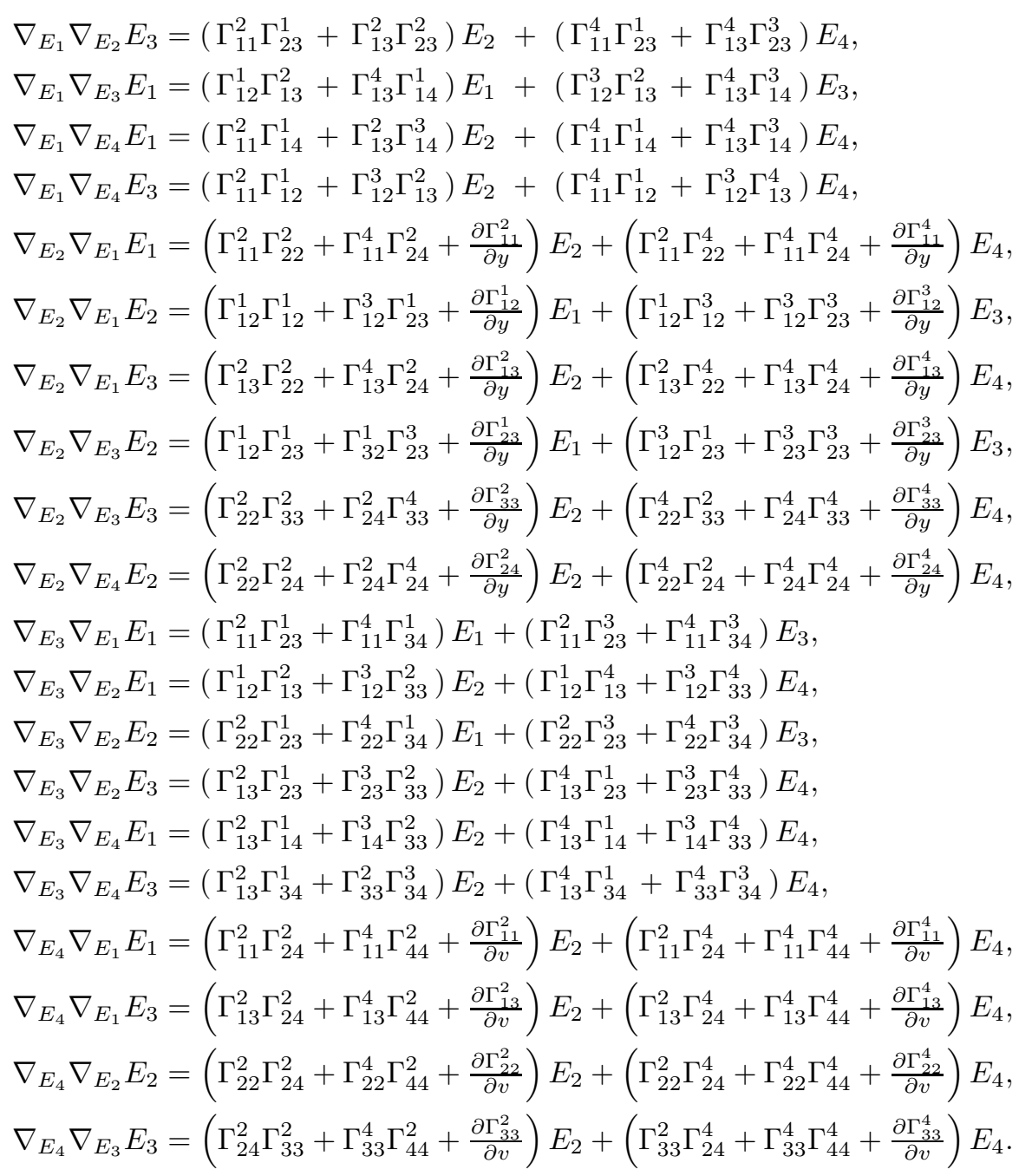

Thus according to Lemma 2.2, Lemma 2.3 and the above formulas, we have

$$
\begin{gathered}
R\left(F_{1}, F_{2}\right) F_{1}=-h_{A}\left\{\left(h_{A} \frac{\partial h_{A}}{\partial y}+\theta_{1}\right) \Gamma_{11}^{2}+h_{A}^{2} \frac{\partial \Gamma_{11}^{2}}{\partial y}\right\} E_{2} \\
-h_{A}\left\{\left(h_{A} \frac{\partial h_{A}}{\partial y}+\theta_{1}\right) \Gamma_{11}^{4}+h_{A}^{2} \frac{\partial \Gamma_{11}^{4}}{\partial y}\right\} E_{4}, \\
R\left(F_{1}, F_{3}\right) F_{1}=h_{A}^{2} h_{C}\left\{\left(\Gamma_{14}^{1} \Gamma_{13}^{4}-\Gamma_{11}^{4} \Gamma_{34}^{1}\right)\right\} E_{1} \\
+h_{A}^{2} h_{C}\left\{\left(\Gamma_{12}^{3} \Gamma_{13}^{2}+\Gamma_{13}^{4} \Gamma_{14}^{3}-\Gamma_{11}^{2} \Gamma_{32}^{3}-\Gamma_{11}^{4} \Gamma_{34}^{3}\right)\right\} E_{3},
\end{gathered}
$$




$$
\begin{aligned}
& R\left(F_{1}, F_{4}\right) F_{1}=h_{A}\left\{h_{A} h_{C}\left(\Gamma_{13}^{2} \Gamma_{14}^{3}-\Gamma_{11}^{4} \Gamma_{44}^{2}-\frac{\partial \Gamma_{11}^{2}}{\partial v}\right)\right. \\
& \left.-h_{A} h_{B} \frac{\partial \Gamma_{11}^{2}}{\partial y}-\left(h_{B} \frac{\partial h_{A}}{\partial y}+h_{C} \frac{\partial h_{A}}{\partial v}+\theta_{2}\right) \Gamma_{11}^{2}\right\} E_{2} \\
& +h_{A}\left\{h_{A} h_{C}\left(\Gamma_{13}^{4} \Gamma_{14}^{3}+\Gamma_{11}^{4} \Gamma_{14}^{1}-\Gamma_{11}^{2} \Gamma_{24}^{2}-\Gamma_{11}^{4} \Gamma_{44}^{4}-\frac{\partial \Gamma_{11}^{4}}{\partial v}\right)\right. \\
& \left.-h_{A} h_{B} \frac{\partial \Gamma_{11}^{4}}{\partial y}-\left(h_{B} \frac{\partial h_{A}}{\partial y}+h_{C} \frac{\partial h_{A}}{\partial v}+\theta_{2}\right) \Gamma_{11}^{4}\right\} E_{4}, \\
& R\left(F_{2}, F_{3}\right) F_{2}=h_{A}\left\{h_{A} h_{C}\left(\Gamma_{23}^{1} \Gamma_{23}^{3}-\Gamma_{22}^{4} \Gamma_{34}^{1}+\frac{\partial \Gamma_{23}^{1}}{\partial y}\right)\right. \\
& \left.+\left(h_{A} h_{B} \frac{\partial \Gamma_{12}^{1}}{\partial y}+h_{A} \frac{\partial h_{B}}{\partial y} \Gamma_{12}^{1}+h_{A} \frac{\partial h_{C}}{\partial y} \Gamma_{23}^{1}-\theta_{3} \Gamma_{12}^{1}-\theta_{4} \Gamma_{23}^{1}\right)\right\} E_{1} \\
& +h_{A}\left\{h_{A} h_{C}\left(\Gamma_{12}^{3} \Gamma_{23}^{1}+\Gamma_{23}^{3} \Gamma_{23}^{3}-\Gamma_{22}^{2} \Gamma_{23}^{3}-\Gamma_{22}^{4} \Gamma_{34}^{3}+\frac{\partial \Gamma_{23}^{3}}{\partial y}\right)\right. \\
& \left.+\left(h_{A} h_{B} \frac{\partial \Gamma_{12}^{3}}{\partial y}+h_{A} \frac{\partial h_{B}}{\partial y} \Gamma_{12}^{3}+h_{A} \frac{\partial h_{C}}{\partial y} \Gamma_{23}^{3}-\theta_{3} \Gamma_{12}^{3}-\theta_{4} \Gamma_{23}^{3}\right)\right\} E_{3}, \\
& R\left(F_{2}, F_{4}\right) F_{2}=\left\{h_{A}^{2} h_{C}\left(\Gamma_{24}^{2} \Gamma_{24}^{4}-\Gamma_{22}^{4} \Gamma_{44}^{2}+\frac{\partial \Gamma_{24}^{2}}{\partial y}-\frac{\partial \Gamma_{22}^{2}}{\partial v}\right)\right. \\
& +h_{A}\left(h_{A} \frac{\partial h_{B}}{\partial y}-h_{B} \frac{\partial h_{A}}{\partial y}-h_{C} \frac{\partial h_{A}}{\partial v}\right) \Gamma_{22}^{2}+h_{A}^{2} \frac{\partial h_{C}}{\partial y} \Gamma_{24}^{2} \\
& +h_{A} \frac{\partial h_{A}}{\partial y} \frac{\partial h_{B}}{\partial y}+h_{A} \frac{\partial h_{C}}{\partial y} \frac{\partial h_{A}}{\partial v}-h_{B}\left(\frac{\partial h_{A}}{\partial y}\right)^{2}-h_{C} \frac{\partial h_{A}}{\partial y} \frac{\partial h_{A}}{\partial v} \\
& \left.-h_{A} \theta_{5} \Gamma_{22}^{2}-h_{A} \theta_{4} \Gamma_{24}^{2}-\theta_{5} \frac{\partial h_{A}}{\partial y}-\theta_{4} \frac{\partial h_{A}}{\partial v}\right\} E_{2} \\
& +\left\{h _ { A } ^ { 2 } h _ { C } \left(\Gamma_{22}^{4} \Gamma_{24}^{2}+\Gamma_{24}^{4} \Gamma_{24}^{4}+\frac{\partial \Gamma_{24}^{4}}{\partial y}-\Gamma_{22}^{2} \Gamma_{24}^{4}-\Gamma_{22}^{4} \Gamma_{44}^{4}\right.\right. \\
& \left.-\frac{\partial \Gamma_{22}^{4}}{\partial v}\right)+h_{A}\left(h_{A} \frac{\partial h_{B}}{\partial y}-h_{B} \frac{\partial h_{A}}{\partial y}-h_{C} \frac{\partial h_{A}}{\partial v}\right) \Gamma_{22}^{4} \\
& \left.+h_{A}^{2} \frac{\partial h_{C}}{\partial y} \Gamma_{24}^{4}-h_{A} \theta_{5} \Gamma_{22}^{4}-h_{A} \theta_{4} \Gamma_{24}^{4}\right\} E_{4}, \\
& R\left(F_{3}, F_{4}\right) F_{3}=-\left\{h_{B}^{2}\left(h_{B} \frac{\partial \Gamma_{11}^{2}}{\partial y}+h_{C} \frac{\partial \Gamma_{11}^{2}}{\partial v}+2 h_{C} \frac{\partial \Gamma_{13}^{2}}{\partial y}\right)\right.
\end{aligned}
$$




$$
\begin{array}{r}
\left.+h_{C}^{2}\left(h_{C} \frac{\partial \Gamma_{33}^{2}}{\partial v}+h_{B} \frac{\partial \Gamma_{33}^{2}}{\partial v}+2 h_{B} \frac{\partial \Gamma_{13}^{2}}{\partial v}\right)\right\} E_{2} \\
-\left\{h_{B}^{2}\left(h_{B} \frac{\partial \Gamma_{11}^{4}}{\partial y}+h_{C} \frac{\partial \Gamma_{11}^{4}}{\partial v}+2 h_{C} \frac{\partial \Gamma_{13}^{4}}{\partial y}\right)\right. \\
\left.+h_{C}^{2}\left(h_{C} \frac{\partial \Gamma_{33}^{4}}{\partial v}+h_{B} \frac{\partial \Gamma_{33}^{4}}{\partial v}+2 h_{B} \frac{\partial \Gamma_{13}^{4}}{\partial v}\right)\right\} E_{4},
\end{array}
$$

where we put

$\theta_{1}:=-\frac{y^{2}\left(2 A y+3 B v^{2}\right)}{2\left(A y+B v^{2}\right)^{2}}, \theta_{2}:=-\frac{B \sqrt{B} y^{\frac{3}{2}} v^{3}}{2 \sqrt{A}\left(A y+B v^{2}\right)^{2}}, \theta_{3}:=\frac{\sqrt{B} y^{\frac{3}{2}} v\left(A y+2 B v^{2}\right)}{2 \sqrt{A}\left(A y+B v^{2}\right)^{2}}$

and

$$
\theta_{4}:=\frac{\sqrt{A} y^{\frac{3}{2}}}{2 \sqrt{B}\left(A y+B v^{2}\right)}, \quad \theta_{5}:=\frac{\sqrt{B} y^{\frac{3}{2}} v}{2 \sqrt{A}\left(A y+B v^{2}\right)} .
$$

Using (2.2), (2.5), (2.7), Lemma 2.1, Lemma 2.2 and the above formulas, we obtain the above sectional curvatures $K_{p}\left(\pi_{i j}\right)$ for $1 \leq i \leq j \leq 4$.

Theorem 3.2. The scalar curvature $r(p)$ of the Siegel-Jacobi space

$$
\left(M, d s_{1,1 ; A, B}^{2}\right)
$$

is

$$
r(p)=-\frac{3}{A} \quad \text { for all } p \in M .
$$

Proof. We recall that the scalar curvature $r(p)$ of $M$ is defined as

$$
r(p):=\sum_{i, j=1}^{4} R\left(F_{i p}, F_{j p}, F_{j p}, F_{i p}\right), \quad p \in M .
$$

We note that the scalar curvature $r(p)$ is independent of the choice of an orthonormal basis of $T_{p}(M)$. Since the following symmetry relations

$$
R(X, Y) Z+R(Y, X) Z=0
$$

hold for all $X, Y, Z \in \mathcal{X}(M)$, we have

$$
\begin{aligned}
r(p)= & -2\left\{R\left(F_{1 p}, F_{2 p}, F_{1 p}, F_{2 p}\right)+R\left(F_{1 p}, F_{3 p}, F_{1 p}, F_{3 p}\right)\right. \\
& +R\left(F_{1 p}, F_{4 p}, F_{1 p}, F_{4 p}\right)+R\left(F_{2 p}, F_{3 p}, F_{2 p}, F_{3 p}\right) \\
& \left.+R\left(F_{2 p}, F_{4 p}, F_{2 p}, F_{4 p}\right)+R\left(F_{3 p}, F_{4 p}, F_{3 p}, F_{4 p}\right)\right\} .
\end{aligned}
$$

Accoding to Theorem 3.1, we obtain

$$
r(p)=-\frac{3}{A}
$$

This completes the proof of the above theorem. 
Remark 3.1. The Poincaré upper half plane $\mathbb{H}_{1}$ is a two dimensional Riemannian manifold with the Poincaré metric

$$
d s_{0}^{2}:=\frac{d x^{2}+d y^{2}}{y^{2}}, \quad z=x+i y \in \mathbb{H}_{1} \text { with } x, y \text { real. }
$$

It is easily seen that the Gaussian curvature of $\left(\mathbb{H}_{1}, d s_{0}^{2}\right)$ is -1 everywhere and $\left(\mathbb{H}_{1}, d s_{0}^{2}\right)$ is an Einstein manifold. Indeed, if we denote by $S_{0}(X, Y)$ the Ricci curvature of $\left(\mathbb{H}_{1}, d s_{0}^{2}\right)$, then we have

$$
S_{0}(X, Y)=-g_{0}(X, Y) \quad \text { for all } X, Y \in \mathcal{X}\left(\mathbb{H}_{1}\right),
$$

where $g_{0}(X, Y)$ is the inner product on the tangent bundle $T\left(\mathbb{H}_{1}\right)$ induced by the Poincaré $d s_{0}^{2}$. But the Siegel-Jacobi space $\left(\mathbb{H}_{1} \times \mathbb{C}, d s_{1,1 ; 1,1}^{2}\right)$ is not an Einstein manifold. In fact, if we denote by $S(X, Y)$ the Ricci curvature of the Siegel-Jacobi space $\left(\mathbb{H}_{1} \times \mathbb{C}, d s_{1,1 ; 1,1}^{2}\right)$, we can see without difficulty that there does not exist a constant $c$ such that

$$
S\left(E_{1}, E_{1}\right)=\operatorname{cg}\left(E_{1}, E_{1}\right)=c g_{11} .
$$

\section{Final remarks}

Let $\mathbb{D}=\{\zeta \in \mathbb{C}|| \zeta \mid<1\}$ be the unit disk in the complex plane. We let

$$
G_{*}^{J}:=\left\{\left(\left(\begin{array}{cc}
p & q \\
\bar{q} & \bar{p}
\end{array}\right),(\xi, \bar{\xi} ; i \kappa)\right) \mid\left(\begin{array}{cc}
p & q \\
\bar{q} & \bar{p}
\end{array}\right) \in S U(1,1), \xi \in \mathbb{C}, \kappa \in \mathbb{R}\right\}
$$

be the Jacobi group equipped with the multiplication law

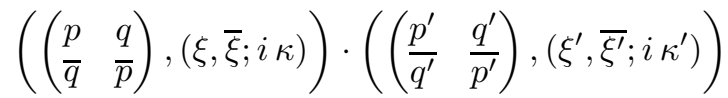

$$
\begin{aligned}
& =\left(\left(\begin{array}{cc}
p & q \\
\bar{q} & \bar{p}
\end{array}\right)\left(\begin{array}{cc}
\frac{p^{\prime}}{q^{\prime}} & \frac{q^{\prime}}{p^{\prime}}
\end{array}\right),\left(\tilde{\xi}+\xi^{\prime}, \tilde{\theta}+\overline{\xi^{\prime}} ; i \kappa+i \kappa^{\prime}+\tilde{\xi}^{t} \bar{\xi}^{\prime}-\tilde{\theta}^{t} \xi^{\prime}\right)\right),
\end{aligned}
$$

where $\tilde{\xi}=p^{\prime} \xi+\bar{q} \bar{\xi}$ and $\tilde{\theta}=q^{\prime} \xi+\overline{p^{\prime}} \bar{\xi}$. Then $G_{*}^{J}$ acts on the Siegel-Jacobi disk $\mathbb{D} \times \mathbb{C}$ transitively by

$$
\left(\left(\begin{array}{cc}
p & q \\
\bar{q} & \bar{p}
\end{array}\right),(\xi, \bar{\xi} ; i \kappa)\right) \cdot(\zeta, \eta)=\left(\frac{p \zeta+q}{\bar{q} \zeta+\bar{p}}, \frac{\eta+\xi \zeta+\bar{\xi}}{\bar{q} \zeta+\bar{p}}\right),
$$

where $\zeta \in \mathbb{D}$ and $\eta \in \mathbb{C}$. According to $(1.2)$, we see that $G_{1,1}^{J}$ acts on $\mathbb{H}_{1} \times \mathbb{C}$ transitively by

$$
\left(\left(\begin{array}{ll}
a & b \\
c & d
\end{array}\right),(\lambda, \mu ; \kappa)\right) \cdot(z, w)=\left(\frac{a z+b}{c z+d}, \frac{w+\lambda z+\mu}{c z+d}\right),
$$

where $\left(\begin{array}{ll}a & b \\ c & d\end{array}\right) \in S L(2, \mathbb{R}), \lambda, \mu, \kappa \in \mathbb{R}, z \in \mathbb{H}_{1}$ and $w \in \mathbb{C}$.

In [15], the author proved that the action (4.1) of $G_{*}^{J}$ on the Siegel-Jacobi disk $\mathbb{D} \times \mathbb{C}$ is compatible with the action (4.2) of $G_{*}^{J}$ on the Siegel-Jacobi space 
$\mathbb{H}_{1} \times \mathbb{C}$ via the partial Cayley tranform $\Phi_{*}: \mathbb{D} \times \mathbb{C} \longrightarrow \mathbb{H}_{1} \times \mathbb{C}$ defined by

$$
\Phi_{*}(\zeta, \eta):=\left(\frac{i(1+\zeta)}{1-\zeta}, \frac{2 i \eta}{1-\zeta}\right), \quad(\zeta, \eta) \in \mathbb{D} \times \mathbb{C}
$$

Precisely, if $g=\left(\left(\begin{array}{ll}a & b \\ c & d\end{array}\right),(\lambda, \mu ; \kappa)\right) \in G_{1,1}^{J}$, we put

$$
g_{*}=\left(\left(\begin{array}{ll}
p & q \\
\bar{q} & \bar{p}
\end{array}\right),\left(\frac{1}{2}(\lambda+i \mu), \frac{1}{2}(\lambda-i \mu) ;-i \frac{\kappa}{2}\right)\right),
$$

where

$$
p=\frac{1}{2}\{(a+d)+i(b-c)\}
$$

and

$$
q=\frac{1}{2}\{(a-d)-i(b+c)\} .
$$

We note that $g_{*}$ is an element of $G_{*}^{J}$. The compatibility condition means that the following condition

$$
g \cdot \Phi_{*}(\zeta, \eta)=\Phi_{*}\left(g_{*} \cdot(\zeta, \eta)\right) \quad \text { for all } g \in G_{1,1}^{J} \text { and }(\zeta, \eta) \in \mathbb{D} \times \mathbb{C}
$$

holds. Using the compatibility condition (4.5), the author [16] proved that for any two positive real numbers $A$ and $B$,

$$
\begin{aligned}
d \tilde{s}_{1,1 ; A, B}^{2}= & 4 A \frac{d \zeta d \bar{\zeta}}{\left(1-|\zeta|^{2}\right)^{2}} \\
& +4 B\left\{\frac{d \eta d \bar{\eta}}{1-|\zeta|^{2}}+\frac{\left(1+|\zeta|^{2}\right)|\eta|^{2}-\bar{\zeta} \eta^{2}-\zeta \bar{\eta}^{2}}{\left(1-|\zeta|^{2}\right)^{3}} d \zeta d \bar{\zeta}\right. \\
& \left.\quad+\frac{\eta \bar{\zeta}-\bar{\eta}}{\left(1-|\zeta|^{2}\right)^{2}} d \zeta d \bar{\eta}+\frac{\bar{\eta} \zeta-\eta}{\left(1-|\zeta|^{2}\right)^{2}} d \bar{\zeta} d \eta\right\}
\end{aligned}
$$

is a Riemannian metric on the Siegel-Jacobi disk $\mathbb{D} \times \mathbb{C}$ which is invariant under the action $(4.1)$ of $G_{*}^{J}$ on $\mathbb{D} \times \mathbb{C}$. According to Theorem 1.4 in [16], we see that the Laplace-Beltrami operator $\widetilde{\Delta}_{1,1 ; A, B}$ of the Siegel-Jacobi disk $\left(\mathbb{D} \times \mathbb{C}, d \tilde{s}_{1,1 ; A, B}^{2}\right)$ is given by

$$
\begin{aligned}
\widetilde{\Delta}_{1,1 ; A, B}=\frac{1}{A}\{ & \left(1-|\zeta|^{2}\right)^{2} \frac{\partial^{2}}{\partial \zeta \partial \bar{\zeta}}+\left(1-|\zeta|^{2}\right)(\eta-\bar{\eta} \zeta) \frac{\partial^{2}}{\partial \zeta \partial \bar{\eta}} \\
& +\left(1-|\zeta|^{2}\right)(\bar{\eta}-\eta \bar{\zeta}) \frac{\partial^{2}}{\partial \bar{\zeta} \partial \eta} \\
& \left.+\left(|\eta|^{2}+|\zeta \eta|^{2}-\bar{\zeta} \eta^{2}-\zeta \bar{\eta}^{2}\right) \frac{\partial^{2}}{\partial \eta \partial \bar{\eta}}\right\} \\
+ & \frac{1}{B}\left(1-|\zeta|^{2}\right) \frac{\partial^{2}}{\partial \eta \partial \bar{\eta}} .
\end{aligned}
$$


Theorem 4.1. The scalar curvature of the Siegel-Jacobi disk $\left(\mathbb{D} \times \mathbb{C}, d \tilde{s}_{1,1 ; A, B}^{2}\right)$ is

$$
r(q)=-\frac{3}{A} \quad \text { for all } q \in \mathbb{D} \times \mathbb{C} .
$$

Proof. The proof follows from Theorem 3.2 and the compatibility condition (4.5).

\section{References}

[1] R. Berndt and R. Schmidt, Elements of the Representation Theory of the Jacobi Group, Progress in Mathematics, 163, Birkhäuser, Basel, 1998.

[2] W. Boothby, An Introduction to Differentiable Manifolds and Riemannian Geometry, Academic Press, 1975.

[3] M. Eichler and D. Zagier, The Theory of Jacobi Forms, Progress in Mathematics, 55, Birkhäuser, Boston, Basel and Stuttgart, 1985.

[4] E. Kähler, Mathematische Werke/Mathematical Works, Edited by R. Berndt and O. Riemenschneider, Walter de Gruyter, Berlin-New York, 2003.

[5] J. Kramer, A geometrical approach to the theory of Jacobi forms, Compositio Math. 79 (1991), no. 1, 1-19.

[6] _ An arithmetic theory of Jacobi forms in higher dimensions, J. Reine Angew. Math. 458 (1995), 157-182.

[7] B. Runge, Theta functions and Siegel-Jacobi forms, Acta Math. 175 (1995), no. 2, 165-196.

[8] M. Spivak, A Comprehensive Introduction to Differential Geometry, Publish or Perish, Inc., Berkeley, Vol. II, 1979.

[9] J.-H. Yang, The Siegel-Jacobi operator, Abh. Math. Sem. Univ. Hamburg 63 (1993), 135-146.

[10] Singular Jacobi forms, Trans. Amer. Math. Soc. 347 (1995), no. 6, 2041-2049.

[11] Construction of vector valued modular forms from Jacobi forms, Canadian J. Math. 47 (1995), no. 6, 1329-1339.

[12] The method of orbits for real Lie groups, Kyungpook Math. J. 42 (2002), no. 2, 199-272.

[13] A note on a fundamental domain for Siegel-Jacobi space, Houston J. Math. 32 (2006), no. 3, 701-712.

[14] Invariant metrics and Laplacians on Siegel-Jacobi space, J. Number Theory 127 (2007), no. 1, 83-102.

[15] - A partial Cayley transform for Siegel-Jacobi disk, J. Korean Math. Soc. 45 (2008), no. 3, 781-794.

[16] Invariant metrics and Laplacians on Siegel-Jacobi disk, Chinese Ann. Math. Ser. B 31 (2010), no. 1, 85-100.

[17] _ Invariant differential operators on Siegel-Jacobi space, arXiv:1107.0509 v1 [math.NT] 4 July 2011.

[18] C. Ziegler, Jacobi forms of higher degree, Abh. Math. Sem. Univ. Hamburg 59 (1989), 191-224.

JAE-HYUN YANG

Department of Mathematics

INHA UNIVERSITY

INCHEON 402-751, KOREA

E-mail address: jhyang@inha.ac.kr 
YOUNG-HOON YONG

Graduate School of Mathematics Education

INHA UNIVERSITY

INCHEON 402-751, KoREA

E-mail address: yyh0302@hanmail.net

Su-Na HuH

Graduate School of Mathematics Education

INHA UNIVERSITY

INCHEON 402-751, KOREA

E-mail address: soonassi@hanmail.net

Jung-HeE Shin

Graduate School of Mathematics Education

INHA UNIVERSITY

INCHEON 402-751, KoREA

E-mail address: sjhee1031@hanmail.net

GiL-Hong Min

Graduate School of Mathematics Education

INHA UNIVERSITY

INCHEON 402-751, KOREA

E-mail address: segeromin@paran.com 